\title{
Holomorphic curves omitting five planes in projective space
}

\author{
Alexandre Eremenko*
}

\begin{abstract}
In 1928 H. Cartan proved an extension of Montel's normality criterion to holomorphic curves in complex projective plane $\mathbf{P}^{2}$. He also conjectured that a similar result is true for holomorphic curves in $\mathbf{P}^{n}$ for any $n$. Recently the author constructed a counterexample to this conjecture for any $n \geq 3$. In this paper we show how to modify Cartan's conjecture so that it becomes true, at least for $n=3$.
\end{abstract}

1. Introduction. A classical theorem of Borel says that any holomorphic mapping $f: \mathbf{C} \rightarrow \mathbf{P}^{n}$ omitting $p=n+2$ hyperplanes in general position must be linearly degenerate - that is the image $f(\mathbf{C})$ must be contained in a hyperplane. To state the theorem more precisely we choose the representation of $\mathbf{P}^{n}$ as the hyperplane in $\mathbf{P}^{n+1}$ defined in homogenous coordinates by the equation $x_{0}+\ldots+x_{n+1}=0$. This representation has the advantage that the $n+2$ omitted hyperplanes can be described by symmetric equations $x_{j}=0,0 \leq j \leq n+1$.

Borel's Theorem. Let $f_{j}$ be entire functions without zeros satisfying

$$
f_{1}+\ldots+f_{p}=0 .
$$

Then there exists a partition of the set of functions $\left\{f_{j}\right\}$ into classes such that all functions in the same class are constant multiples of each other and the sum of the functions in each class is zero.

${ }^{*}$ A part of this work was done when the author was visiting Université de Paris-Sud (Orsay). This work was also supported by NSF grant DMS-9500636 
The case $p=3$ of Borel's theorem is nothing but the Little Picard Theorem. Indeed, to say that an entire function $f$ omits 0 and 1 is the same as to say $f+g-1=0$, where $f$ and $g$ have no zeros.

According to the so-called Bloch Principle, to Borel's theorem there should correspond a normality criterion, just as Montel's theorem corresponds to Picard's theorem. We refer to $[2,6]$ and $[7]$ for general discussion of this heuristic principle. But, as Bloch remarks in [1], cf. [6, p. 224] it is not at all clear at first sight what this normality criterion should be.

Set $D(a, r)=\{z \in \mathbf{C}:|z|<r\}, D(r)=D(0, r)$ and let $U$ denotes the set of holomorphic functions $g$ in $D(1)$ such that $g(z) \neq 0, z \in D(1)$. Such functions are called units. We are going to study infinite families $F=\{f\}$ of $p$-tuples $f=\left(f_{1}, \ldots, f_{p}\right), f_{j} \in U$, satisfying the equation

$$
f_{1}+\ldots+f_{p}=0 .
$$

Given such a family $F$ let $\mathcal{F}$ denotes the filter formed by complements of finite subsets of $F$.

A subset of indices $S \subset\{1, \ldots, p\}$ is called a C-class if (i) there exists $k \in S$ such that $f_{j} / f_{k}$ are uniformly bounded on compacta as $f \rightarrow \mathcal{F}$ for all $j \in S$ and

(ii) $\sum_{j \in S} f_{j} / f_{k} \rightarrow 0$ as $f \rightarrow \mathcal{F}$, uniformly on compacta.

Notice that by (ii) every C-class contains at least 2 elements.

Cartan's conjecture. Given a family $F$ of p-tuples of units satisfying (1) there exists an infinite subfamily $L \subset F$ such that for $f \in L$ the set of indices $\{1, \ldots, p\}$ can be partitioned into $C$-classes.

For $p=3$ this is nothing else but Montel's normality criterion. The conjecture was stated by Cartan in [3], where he proved

Cartan's Theorem. Given a family $F$ of p-tuples of units satisfying (1) there exists an infinite subfamily $L \subset F$ such that for $f \in L$ one of the following holds:

a) the full set of indices $\{1, \ldots, p\}$ forms a $C$-class

or

b) there exist at least two disjoint C-classes. 
This theorem implies Cartan's conjecture for $p=4$ because if the case b) occurs then two C-classes make a partition of the set of indices. When $p \geq 5$ Cartan's theorem falls short of proving his conjecture because in the case b) there may not be enough C-classes to make a partition of $\{1, \ldots, p\}$. On [3, pp. 69-70] Cartan discusses the hypothetical case when $p=5$ and there are only two C-classes each containing two elements but the remaining index does not belong to any C-class. He concludes that constructing such an example would be difficult.

Such example has been recently constructed in [4]. A simplified version will be given in section 4. Actually this example shows that Cartan's conjecture fails even if we replace condition (ii) in the definition of C-class by a weaker condition that every C-class contains at least two elements. Examination of the example as well as our strong belief in Bloch's Principle suggest the following

Modified Conjecture. Let $F$ be an infinite family of p-tuples of units in $D(1)$ satisfying (1). Then there exists an infinite subfamily $L \subset F$ such that for $f \in L$ the set of indices can be partitioned into C-classes in the disk $D\left(r_{p}\right)$ where $0<r_{p}<1$ and $r_{p}$ depends only on $p$.

It will follow from this conjecture that in any hyperbolic disk of sufficiently small radius the partition of the set of indices into C-classes is possible.

We can prove this Modified Conjecture only for $p=5$. The proof given in section 3 is based on the the same techniques used by Bloch and Cartan, that is Nevanlinna theory and estimates of potentials. A very good reference is [6]. The new ingredient is an elementary lemma from potential theory contained in section 2 .

The author thanks D. Drasin, B. Korenblum and the referee for valuable suggestions.

\section{An auxilliary result on harmonic functions}

Lemma 1 Let $u_{1}$ and $u_{2}$ be harmonic functions in the disk $D\left(z_{0}, r\right)$. Denote by $u_{+}$the least harmonic majorant of $u_{1} \vee u_{2}$ and by $u_{-}$the greatest harmonic minorant of $u_{1} \wedge u_{2}$. If $u_{+} \geq 0$ and $u_{-}\left(z_{0}\right)+\delta u_{+}\left(z_{0}\right) \geq 0$ for some $\delta, 0<$ $\delta<1$, then one of the functions $u_{1}, u_{2}$ is non-negative in $D\left(z_{0}\right.$,ar $)$, where 
$a=a(\delta)$ is given by

$$
a(\delta)=\frac{\sqrt{2}-\sqrt{1+\delta}}{\sqrt{2}+\sqrt{1+\delta}} .
$$

Furthermore, we actually have $u_{i}(z) \geq \epsilon u_{+}\left(z_{0}\right)$ for $i=1$ or 2 in the disk $D\left(z_{0}, a^{\prime} r\right), a^{\prime}<a(\delta)$, for some $\epsilon$ depending only on $a^{\prime}$.

Remarks. We have $a(0)=3-2 \sqrt{2} \approx .1716$. It seems interesting to determine the largest value of $a(\delta)$ for which the Lemma is true, at least when $\delta=0$. It is plausible that the extreme functions when $\delta=0$ are

$$
u_{1}(z)=\Re \frac{(1+z)\left(z^{2}-4 z+1\right)}{(1-z)\left(1+z^{2}\right)} \quad \text { and } \quad u_{2}(z)=u_{1}(-z) .
$$

This example shows that $a(0) \leq 2-\sqrt{3} \approx .268$.

Proof of Lemma 1. It is enough to consider the case when $r=1$ and $z_{0}=0$.

We always denote by $\vee$ and $\wedge$ the pointwise maximum and minimum of functions respectively. When $|z|=1$ we have $u_{+}(z)=\left(u_{1} \vee u_{2}\right)(z)$ and $u_{-}(z)=\left(u_{1} \wedge u_{2}\right)(z)$. Thus

$$
u_{1}+u_{2}=u_{+}+u_{-}
$$

so the condition $u_{-}(0)+\delta u_{+}(0) \geq 0$ combined with (3) implies

$$
u_{1}(0)+u_{2}(0) \geq(1-\delta) u_{+}(0) .
$$

It follows that one of the numbers $u_{1}(0), u_{2}(0)$ is at least $(1-\delta) u_{+}(0) / 2$. Suppose that

$$
u_{1}(0) \geq \frac{1-\delta}{2} u_{+}(0)
$$

Applying Harnack's inequality to the positive harmonic function $u_{+}$we obtain

$$
u_{+}(z) \geq \frac{1-r}{1+r} u_{+}(0), \quad|z| \leq r
$$

On the other hand, $u_{+}-u_{1}$ is also a positive harmonic function, whose value at 0 is at most $(1+\delta) u_{+}(0) / 2$, in view of (4). Thus Harnack's inequality implies

$$
\left(u_{+}-u_{1}\right)(z) \leq \frac{(1+\delta)(1+r)}{2(1-r)} u_{+}(0), \quad|z| \leq r .
$$


Combining (5) and (6), we obtain for $|z| \leq r$

$u_{1}(z) \geq\left(\frac{1-r}{1+r}-\frac{(1+\delta)(1+r)}{2(1-r)}\right) u_{+}(0)=\frac{2(1-r)^{2}-(1+\delta)(1+r)^{2}}{2\left(1-r^{2}\right)} u_{+}(0)$.

The last expression is positive when $r<a(\delta)$, where $a(\delta)$ is given by $(2)$.

\section{Proof of the Modified Conjecture for $p=5$.}

In view of Cartan's theorem we may assume that $\{1,3\}$ and $\{2,4\}$ are C-classes (in the full unit disk). Furthermore we may assume that $f_{5}=-1$. Thus we have

$$
f_{1}+f_{2}+f_{3}+f_{4}=1
$$

and by (ii) in the definition of a C-class

$$
f_{3} / f_{1} \rightarrow-1 \text { and } f_{4} / f_{2} \rightarrow-1, \quad \text { as } \quad f \rightarrow \mathcal{F}
$$

uniformly on compacta in $|z|<1$. Our goal is to show that either $f_{5} / f_{1}$ or $f_{5} / f_{2}$ tends to zero uniformly on compacta in $|z|<r^{*}=2^{-8}$; that is the index 5 can be added to one of the C-classes which already exist. In other words we want to show that one of the functions $f_{1}$ or $f_{2}$ tends to infinity uniformly on compacta in $|z|<r^{*}$.

Set $g_{1}=f_{1}+f_{3}, g_{2}=f_{2}+f_{4}$ and $g=g_{1}^{\prime}$ (derivative), so that by (7)

$$
g_{1}+g_{2}=1
$$

and

$$
g_{1}^{\prime}=-g_{2}^{\prime}=g
$$

We conclude from (8) that

$$
f_{1} / g_{1}=\left(1+f_{3} / f_{1}\right)^{-1} \rightarrow \infty, \quad f \rightarrow \mathcal{F}
$$

and similarly

$$
f_{2} / g_{2} \rightarrow \infty, \quad f \rightarrow \mathcal{F}
$$

uniformly on compacta in $|z|<1$.

Now it follows from (9) that

$$
\log ^{+}\left|g_{1}\right|=\log ^{+}\left|1-g_{2}\right| \leq \log ^{+}\left|g_{2}\right|+\log 2
$$


and similarly $\log ^{+}\left|g_{2}\right| \leq \log ^{+}\left|g_{1}\right|+\log 2$. Thus

$$
\left|\log ^{+}\right| g_{1}\left|-\log ^{+}\right| g_{2}|| \leq \log 2 .
$$

Again from (9) we conclude that

$$
\left|g_{1}\right| \vee\left|g_{2}\right| \geq 1 / 2,
$$

so we may assume without loss of generality that

$$
\left|g_{1}(0)\right| \geq 1 / 2 .
$$

Now we put $r^{*}=2^{-8}$ and consider three cases.

Case 1.

$$
\left|g_{1}(z)\right| \leq 2 e^{e} \text { for }|z| \leq r^{*} .
$$

We apply Cartan's lemma [6, Ch. VIII, $\S 3]$ to estimate $\left|g_{1}\right|$ from below, using (15) and (16). For any given $\epsilon>0$ we have

$$
\left|g_{1}(z)\right| \geq C(\epsilon) \text { for }|z|=t
$$

with some $t \in\left[r^{*}-\epsilon, r^{*}\right]$. So $\left|f_{1}(z)\right| \rightarrow \infty$ when $|z|=t$ in view of (11), and hence, by the Minimum Principle, $f_{1}(z) \rightarrow \infty$ uniformly in $|z| \leq r^{*}-\epsilon$.

Case 2. Now we assume that

$$
\left|g_{1}\left(z_{0}\right)\right| \geq 2 e^{e} \quad \text { for some } z_{0},\left|z_{0}\right| \leq r^{*},
$$

but $|g(z)| \leq 1$ for all $z$ in the disk $|z| \leq r^{*}$.

Then we integrate

$$
g_{1}(z)=g_{1}\left(z_{0}\right)+\int_{z_{0}}^{z} g(\zeta) d \zeta
$$

and obtain $\left|g_{1}(z)\right| \geq 1,|z| \leq r^{*}$. Again (11) concludes the proof in this case.

Case 3. It remains to consider the possibility that there are points $z_{0}$ and $z_{1}$ in the disk $|z| \leq r^{*}$ such that

$$
\left|g_{1}\left(z_{0}\right)\right|>2 e^{e}
$$


and

$$
\left|g\left(z_{1}\right)\right| \geq 1
$$

In view of (13) and (17) we have

$$
\left|g_{2}\left(z_{0}\right)\right| \geq e^{e}
$$

Inequalities (11) and (17) imply

$$
f_{1}\left(z_{0}\right) \rightarrow \infty
$$

For each $f \in F$ we fix reference points $z_{0}$ and $z_{1}$ in $D\left(r^{*}\right)$ satisfying (17) and (18)

Our plan is the following. We are going to apply Lemma 1 to the harmonic functions $u_{1}=\log \left|f_{1}\right|$ and $u_{2}=\log \left|f_{2}\right|$ in an appropriately chosen disk $D\left(z_{0}, r\right)$, with $r>1 / 2$. The least harmonic majorant of $u_{1} \vee u_{2}$ is positive by (11), (12) and (14). We need an estimate for the greatest harmonic minorant $u_{-}$of the function $u_{1} \wedge u_{2}$ at the point $z_{0}$ from below. This is the same as the average of $u_{-}$over the circle $\left|z-z_{0}\right|=r$. To estimate this average from below we will use the derivative $g$ and the subharmonic function $w=\log |g|$. We will show that (up to a small error term) $w$ is a subharmonic minorant for $\log \left|g_{1}\right| \wedge \log \left|g_{2}\right|<u_{1} \wedge u_{2}$, and thus $w\left(z_{0}\right)$ is a minorant for $u_{-}\left(z_{0}\right)$. However instead of a lower estimate of $w$ at $z_{0}$ we only have an estimate at a nearby point $z_{1}$ (see (18)). We will handle this with the help of Lemma 3. Now we go into details.

For a holomorphic function $h$ in the unit disk and positive number $r<$ $1-r^{*}$ we define

$$
m_{z_{0}}(r, h)=\int_{-\pi}^{\pi} \log ^{+}\left|h\left(z_{0}+r e^{i \theta}\right)\right| \frac{d \theta}{2 \pi}, \quad\left|z_{0}\right|<r^{*} .
$$

Since $\log ^{+}|h|$ is subharmonic, $m_{z_{0}}(r, h)$ increases with $r$. We will omit the index $z_{0}$ in this notation with understanding that the point $z_{0}$ specified above is always used. In what follows we use the notation $C_{k}$ for absolute constants (they may be different in each occurence). We need the Lemma on the Logarithmic Derivative. It is convenient to start with the formulation as in [5, Sect. 2.2.2]: for holomorphic functions $g_{i}$ we have for $1 / 2<r<R<1-r^{*}$

$$
m\left(r, g / g_{i}\right) \leq C_{1}+C_{2} \log m\left(R, g_{i}\right)+C_{3} \log \frac{1}{R-r}+C_{4} \log ^{+} \log ^{+} \frac{1}{\left|g_{i}\left(z_{0}\right)\right|}
$$


In view of (17) and (19) the last term can be omitted. We also need to eliminate the term with $\log (R-r)$. This can be done with the following lemma which goes back to E. Borel (see, for example [6, Ch. VIII, Lemma $1.4])$.

Lemma 2 Let $S \geq 0$ be an increasing function on $[0, b], b>0$ and $\gamma>0$. Then there exists a subset $E \subset[0, b]$ of measure at most $2 e^{-S(0) / \gamma}$, such that

$$
S\left(r+e^{-S(r) / \gamma}\right) \leq S(r)+\gamma \log 2, \quad r \notin E .
$$

We choose $S(r)=\log m\left(r, g_{i}\right)$ (so that $S(0) \geq 1$ by (17) and (19)), $\gamma=S(0) /(3 \log 2)$ and put $R=r+e^{-S(r) / \gamma}$ in the Lemma on Logarithmic Derivative. The exceptional set $E$ in Lemma 2 has measure at most 1/4, and the Lemma on Logarithmic Derivative becomes: there exists $r$,

$$
1 / 2=2^{7} r^{*}<r<1-r^{*}
$$

such that

$$
m\left(r, g / g_{i}\right) \leq C_{1}+C_{2} \log m\left(r, g_{i}\right), \quad i=1,2 ;
$$

where $C_{1}$ and $C_{2}$ are absolute constants. We fix this $r$ satisfying (21) and (22) until the end of the proof. (Of course $r$, as well as $z_{0}$ and $z_{1}$, depends on $f$.)

Denote by $u_{+}$the least harmonic majorant of $\log \left|f_{1}\right| \vee \log \left|f_{2}\right|$ in the disk $\left|z-z_{0}\right|<r$. Then by (11), (12) and (14)

$$
u_{+} \rightarrow+\infty, \quad f \rightarrow \mathcal{F} .
$$

uniformly in $D\left(z_{0}, r\right)$. Using (11), (12) positivity and harmonicity of $u_{+}$we obtain

$$
m\left(r, g_{i}\right) \leq m\left(r, f_{i}\right) \leq \int_{-\pi}^{\pi} u_{+}\left(z_{0}+r e^{i \theta}\right) \frac{d \theta}{2 \pi}=u_{+}\left(z_{0}\right), \quad i=1,2 .
$$

Thus (22), (24) and (23) imply for $i=1,2$

$$
m\left(r, g / g_{i}\right) \leq C_{1}+C_{2} \log m\left(r, g_{i}\right) \leq C_{1}+C_{2} \log u_{+}\left(z_{0}\right) \leq o\left(u_{+}\left(z_{0}\right)\right)
$$

as $f \rightarrow \mathcal{F}$. It follows from (24) and (25) that

$$
m(r, g) \leq m\left(r, g_{1}\right)+m\left(r, g / g_{1}\right) \leq(1+o(1)) u_{+}\left(z_{0}\right), \quad f \rightarrow \mathcal{F} .
$$

Now we need the following 
Lemma 3 Let $g$ be an analytic function in the disk $\left|z-z_{0}\right| \leq r$ and suppose that $\left|g\left(z_{1}\right)\right| \geq 1$ for some $z_{1} \in D\left(z_{0}, r\right)$. Then

$$
\int_{-\pi}^{\pi} \log \left|g\left(z_{0}+r e^{i \theta}\right)\right| \frac{d \theta}{2 \pi}+\delta m(r, g) \geq 0
$$

where $\delta=4 r\left|z_{0}-z_{1}\right| /\left(r-\left|z_{0}-z_{1}\right|\right)^{2}$.

Proof. Assume without loss of generality that $z_{0}=0$ and put $\left|z_{1}\right|=t$. Then by Poisson's formula

$$
\begin{gathered}
0 \leq \log \left|g\left(z_{1}\right)\right| \\
\leq \frac{r+t}{r-t} \int_{-\pi}^{\pi} \log ^{+}\left|g\left(r e^{i \theta}\right)\right| \frac{d \theta}{2 \pi}-\frac{r-t}{r+t} \int_{-\pi}^{\pi} \log ^{-}\left|g\left(r e^{i \theta}\right)\right| \frac{d \theta}{2 \pi} \\
=\frac{r-t}{r+t} \int_{-\pi}^{\pi} \log \left|g\left(r e^{i \theta}\right)\right| \frac{d \theta}{2 \pi}+\frac{4 r t}{r^{2}-t^{2}} m(r, g) .
\end{gathered}
$$

This proves the lemma.

In our situation we have $\left|z_{0}-z_{1}\right| \leq 2 r^{*}=2^{-7}$, so by (21) the number $\delta$ from Lemma 3 has the following bound:

$$
\delta \leq \frac{8 r r^{*}}{\left(r-2 r^{*}\right)^{2}} \leq \frac{16 r^{*}}{r} \leq 2^{-3}
$$

We apply Lemma 3 to our function $g$ and use (18) (27) and (26) to obtain

$$
\int_{-\pi}^{\pi} \log \left|g\left(z_{0}+r e^{i \theta}\right)\right| \frac{d \theta}{2 \pi}+\left(\frac{1}{8}+o(1)\right) u_{+}\left(z_{0}\right) \geq 0, \quad f \rightarrow \mathcal{F} .
$$

Finally we estimate $|g|$ from above:

$$
\log |g| \leq \log \left|g_{i}\right|+\log ^{+}\left|g / g_{i}\right|, \quad i=1,2 .
$$

These inequalities together with (11) and (12) imply

$$
\begin{gathered}
\log |g| \leq \log \left|g_{1}\right| \wedge \log \left|g_{2}\right|+\log ^{+}\left|g / g_{1}\right|+\log ^{+}\left|g / g_{2}\right| \\
\leq \log \left|f_{1}\right| \wedge \log \left|f_{2}\right|+\log ^{+}\left|g / g_{1}\right|+\log ^{+}\left|g / g_{2}\right| .
\end{gathered}
$$


We integrate this inequality over the circle $\left|z-z_{0}\right|=r$ and use (25) to estimate the integrals involving logarithmic derivatives:

$$
\int_{-\pi}^{\pi}\left(\log \left|f_{1}\right| \wedge \log \left|f_{2}\right|\right)\left(z_{0}+r e^{i \theta}\right) \frac{d \theta}{2 \pi} \geq \int_{-\pi}^{\pi} \log \left|g\left(z_{0}+r e^{i \theta}\right)\right| \frac{d \theta}{2 \pi}+o\left(u_{+}(0)\right),
$$

which with (28) gives

$$
\left.\int_{-\pi}^{\pi}\left(\log \left|f_{1}\right| \wedge \log \left|f_{2}\right|\right)\left(z_{0}+r e^{i \theta}\right) \frac{d \theta}{2 \pi}+\left(\frac{1}{8}+o(1)\right)\right) u_{+}\left(z_{0}\right) \geq 0, \quad f \rightarrow \mathcal{F}
$$

so we are in position to apply Lemma 1 with $\delta<1 / 8$. Using this Lemma we conclude that either $\log \left|f_{1}\right|$ or $\log \left|f_{2}\right|$ tends to infinity in the disk

$$
D\left(z_{0}, a^{\prime} r\right)
$$

where $a^{\prime}<a(1 / 8)$ and $r>1 / 2$ (see (21)). A simple computation with (2) shows that $a(1 / 8)>1 / 7$, so we may take $a^{\prime}=1 / 10$ and then $a^{\prime} r>1 / 20$. We also have $\left|z_{0}\right| \leq r^{*}=2^{-8}$, thus the disk (29) contains $D\left(0, r^{*}\right)$ and this finishes the proof.

4. A counterexample to Cartan's conjecture. For $|z|<1$ and positive integer $n$ put

$$
g_{1, n}=\sqrt{n} \int_{-1}^{z} e^{-n \zeta^{2}} d \zeta
$$

and

$$
g_{2, n}=g_{1, n}(-z)=\sqrt{n} \int_{z}^{1} e^{-n \zeta^{2}} d \zeta
$$

so that

$$
g_{1, n}+g_{2, n}=\sqrt{n} \int_{-1}^{1} e^{-n \zeta^{2}} d \zeta=c_{n}=\sqrt{\pi}+o(1), \quad n \rightarrow \infty
$$

Elementary estimates show that

$$
\left|g_{i, n}(z)\right| \leq 2 \sqrt{n} e^{n}, \quad|z|<1, \quad i=1,2
$$

and

$$
\left|g_{1, n}(z)\right| \leq \sqrt{n} e^{-n / 2}, \quad \Re z<-\frac{\sqrt{3}}{2},|z|<1
$$


Thus if we put

$$
f_{1, n}(z)=\exp \{n(14(z+1)-1 / 3)\}
$$

and $f_{2, n}(z)=f_{1, n}(-z)$ then

$$
g_{1, n}(z)=o\left(f_{1, n}(z)\right), \quad n \rightarrow \infty
$$

uniformly in $D(1)$, and

$$
g_{2, n}(z)=o\left(f_{2, n}(z)\right), \quad n \rightarrow \infty
$$

uniformly in $D(1)$.

Evidently $f_{1, n}$ and $f_{2, n}$ are units. So are $f_{3, n}:=-f_{1, n}+g_{1, n}$ and $f_{4, n}:=$ $-f_{2, n}+g_{2, n}$ in view of (31) and (32). If we put $f_{5, n}:=-c_{n}$ then it is also a unit (just a constant) and

$$
f_{1, n}+f_{2, n}+f_{3, n}+f_{4, n}+f_{5, n}=0
$$

in view of $(30)$.

It remains to notice that $f_{5, n}$ cannot belong to any C-class. Indeed, none of the sequences $f_{i, n}, 1 \leq i \leq 4$ is bounded from above or away from zero on compacta in $D(1)$. Thus by (30) none of the quotients $f_{i, n} / f_{5, n}$ can be normal in $D(1)$.

Addition of April 24, 1996. P. M. Tamrazov constructed an example which shows that the expression (2) gives the largest value of $a(\delta)$ for which the statement of Lemma 1 is true, for every $\delta \in(0,1)$. Thus Lemma 1 gives the best possible estimate and the conjecture stated in the Remark after Lemma 1 is wrong.

We describe the example with P. M. Tamrazov's permission. Let

$$
P(z, t)=\Re \frac{e^{i t}+z}{e^{i t}-z}
$$

be the Poisson kernel. Put

$$
u_{\epsilon}=P(., \pi)-\frac{1+\delta}{4}(P(., \epsilon)+P(.,-\epsilon)) .
$$

A straightforward computation shows that $u_{\epsilon}$ has a positive zero which tends to $a(\delta)$ as $\epsilon \rightarrow 0$, where $a(\delta)$ is given by (2). On the other hand, if $\epsilon_{1}$ and $\epsilon_{2}$ 
are two different numbers on $(0, \pi)$ then $u_{\epsilon_{1}}$ and $u_{\epsilon_{2}}$ satisfy all conditions of Lemma 1 because in this case $u_{+}=P(., \pi)>0$ and

$u_{-}(0)=P(0, \pi)-\frac{(1+\delta)}{4}\left(P\left(0, \epsilon_{1}\right)+P\left(0,-\epsilon_{1}\right)+P\left(0, \epsilon_{2}\right)+P\left(0,-\epsilon_{2}\right)\right)=-\delta$,

so $\delta u_{+}(0)+u_{-}(0)=0$.

\section{References}

[1] André Bloch, Sur les systèmes de fonctions holomorphes a variétés linéaires lacunaires, Ann. École Norm. Supèr., 43 (1926), 309-362.

[2] André Bloch, La conception actuelle de la théorie des fonctions entières et méromorphes, Enseignement mathématique, 1926.

[3] Henri Cartan, Sur les systèmes de fonctions holomorphes à variétés linéaires lacunaires et leurs applications, Ann. École Normale Supèr., 45 (1928), 255-346.

[4] Alexandre Eremenko, A counterexample to Cartan's conjecture on holomorphic curves omitting hyperplanes, Preprint, Purdue University, April, 1995, Accepted in Proc. AMS.

[5] Walter K. Hayman, Meromorphic Functions, Clarendon press, Oxford, 1964.

[6] Serge Lang, Introduction to Complex Hyperbolic Spaces, Springer-Verlag, NY, 1987.

[7] Lawrence Zalcman, A heuristic principle in complex function theory, Amer. Math. Monthly, 82 (1975), 813-817.

Purdue University West Lafayette IN 47907

e-mail: eremenko@math.purdue.edu 